\title{
PHLEGMON PERI-AMYGDALIEN ASPECTS DIAGNOSTIQUES ET THÉRAPEUTIQUES
}

\author{
M. MAÂMOURI, R. B. HAMOUDA, S. MANSOUR, A. CHORFA, I. CHTIOUI, K. BOUZAIDI* \\ SERVICE D'ORL ET DE CHIRURGIE CERVICO-FACIALE \\ *SERVICE DE RADIOLOGIE \\ CHU M. T. MAÂMOURI - NABEUL.
}

\begin{abstract}
RESUME
OBJECTIFS : Le phlegmon péri-amygdalien (PPA) est une complication suppurée de la face et du cou souvent rencontrée en urgence ORL. II peut poser des problèmes diagnostiques et thérapeutiques et mettre en jeu le pronostic vital. L'objectif de cette étude est de préciser les particularités épidémiologiques et cliniques de cette affection et de revoir les possibilités thérapeutiques à partir d'une revue de la littérature.

Matériel et méthode : Cette étude est rétrospective portant sur 75 cas de PPA, colligés au service d'ORL et de Chirurgie cervico-faciale de l'Hôpital de Nabeul sur une période de 7 ans (janvier 2001 à décembre 2007). L'étude des dossiers nous a permis de relever toutes les données cliniques et thérapeutiques. Notre recul est de deux ans.

Résultats : L'âge moyen de nos patients était de 26 ans avec un sex-ratio de 1. La triade classique du PPA était complète dans $49 \%$ des cas. Tous les patients ont fait l'objet d'une ponction. Celle-ci était positive dans $74 \%$. Quatre vingt douze pour cent des malades ont bénéficié d'un drainage chirurgical. Un germe a été identifié dans 16 cas avec prédominance du streptocoque $\beta$ hémolytique (13 cas). La guérison clinique et biologique a été constatée en moyenne au dixième jour. Une amygdalectomie à froid a été réalisée chez 57 patients après un intervalle de 2 à 6 mois. Aucune amygdalectomie à chaud n'a été réalisée.

Conclusion : Le Phlegmon péri-amygdalien est une urgence fréquente en ORL. Le diagnostic est clinique. La ponction réalisée dans tous les cas permet de confirmer le diagnostic dans les formes incomplètes. Le traitement est médico-chirurgical.
\end{abstract}

Mots clés : Phlegmon péri-amygdalien, Diagnostic, Traitement.

Introduction: The Peritonsillar abscesse (PTA) is a suppurated complication of the face and the neck often met as a matter of urgency ENT. He can raise diagnostic and therapeutic problems and be life-threatening. The objective of this study is to specify the epidemiological and clinical particularities of this affection and to revise the therapeutic possibilities from a review of the literature.

PATIENTS AND METHODS: This retrospective study concerning 75 patients hospitalized for PTA in the Department of Otolaryngology-Head and Neck Surgery of the Hospital of Nabeul over a period of 7 years (in January 2001 to December 2007). The study of files allowed us to find all the clinical and therapeutic data. Our drop is of two years.

RESULTS : The average age of our patients was of 26 years with a sex ratio of 1 . The classic set of three of the PTA was complete only in $49 \%$ of the cases. All the patients were the object of a needle aspiration, it was positive in $74 \%$ among which $92 \%$ benefited from a drainage under local anesthetic. A germ was identified in 16 cases with a Streptococcus $\beta$ hemolytic group A in 13 cases. The clinical and biological cure was noticed on average in the tenth day. Forty-seven patients underwent tonsillectomy at a later date after an interval from 2 to 6 months. No emergency tonsillectomy was realized.

CONCLUSION : The Peritonsillar abscesses is a frequent urgency in ENT. The diagnostic is clinical. The practicable of needle aspiration in every case allows to confirm the diagnosis in the incomplete forms. The treatment is medical and surgical.

KEYWORDS : Peritonsillar abscesses, diagnostic, treatment

\section{INTRODUCTION}

Le phlegmon péri-amygdalien (PPA) correspond au développement d'une collection purulente dans l'espace péri amygdalien (1). II s'agit d'une urgence ORL fréquente qui peut poser des problèmes diagnostiques et thérapeutiques. Non traité à temps, il peut évoluer vers de graves complications pouvant mettre en jeu le pronostic vital $(2,3)$.
L'objectif de cette étude est de préciser les particularités épidémiologiques et cliniques de cette affection et d'en revoir les possibilités thérapeutiques à partir d'une revue de la littérature.

\section{MATÉRIEL ET MÉTHODES}

Notre étude est rétrospective. Elle porte sur 75 cas de 
PPA, hospitalisés et traités au service ORL et de Chirurgie cervico-faciale de l'Hôpital de Nabeul sur une période de 7 ans (de janvier 2001 à décembre 2007).

Pour chaque malade, nous avons recueilli des dossiers le maximum de données cliniques et para-cliniques. L'interrogatoire a permis de noter, outre les antécédents pathologiques, les circonstances de survenue de l'infection, les plaintes et la prise de médicaments. L'examen de l'oropharynx a été complété, en cas de trismus très serré, par l'endoscope rigide. La palpation des aires ganglionnaires cervicales, l'évaluation de l'état général (température, état d'hydratation chez l'enfant), la recherche de complications associées ont été systématiques. Un bilan biologique (comprenant toujours une NFS et une VS) a toujours été demandé.

Le dosage de la CRP n'a pas toujours été possible. Jamais un examen radiologique n'a été jugé nécessaire. Nous avons pratiqué une ponction exploratrice en plein bombement dans le pilier antérieur à tous nos malades. Un examen bactériologique a été demandé chaque fois que du pus a été retiré.

Tous les patients ont bénéficié d'une antibiothérapie par voie intra veineuse associée à une (ou plusieurs) ponction(s) évacuatrice(s) ou à un drainage par incision. La guérison a été jugée sur l'amélioration clinique et la disparation du syndrome inflammatoire biologique.

L'évolution a été étudiée avec un recul moyen de deux ans.

La saisie et l'analyse des résultats ont été réalisées par le programme Microsoft Office Excel.

\section{RESULTATS}

Les PPA représentent $82 \%$ des 88 suppurations péripharyngées colligées dans notre service.

II s'agissait de : 75 PPA, 5 suppurations préstyliennes (SPS), 5 suppurations retrostyliennes (SRS) et 3 suppurations retro pharyngées (SRP).

L'âge moyen était de 26 ans avec des extrêmes allant de 4 à 69 ans. Le sex-ratio était de 1 .

Les enfants de moins de 16 ans représentaient $12 \%$ de nos malades. La notion d'angines à répétition a été retrouvée dans $24 \%$ des cas. Une automédication par antibiothérapie avant l'admission a été notée dans $68 \%$ des cas.

Le délai moyen entre l'apparition du premier symptôme et la première consultation a été de 4 jours, avec des extrêmes de 24 heures et de 7 jours (Tableau I).

\begin{tabular}{|l|c|c|c|c|}
\hline Signes fonctionnels & Fièvre & Odynophagie & Otalgie réflexe & Dyspnée inspiratoire \\
\hline Pourcentage & 84 & 100 & 24 & 4 \\
\hline
\end{tabular}

Tableau I : Signes fonctionnels

Le trismus n'était présent que dans $56 \%$ des cas. Il existait un bombement du pilier antérieur du côté atteint et une déviation de la luette du côté opposé au phlegmon dans $100 \%$ des cas. L'œdème de la luette a été constaté dans $58 \%$ des cas.

La triade classique associant ces trois signes à savoir bombement du pilier antérieur, œdème de la luette et trismus était complète dans près de la moitié de nos cas (49\%) (Figure 1).

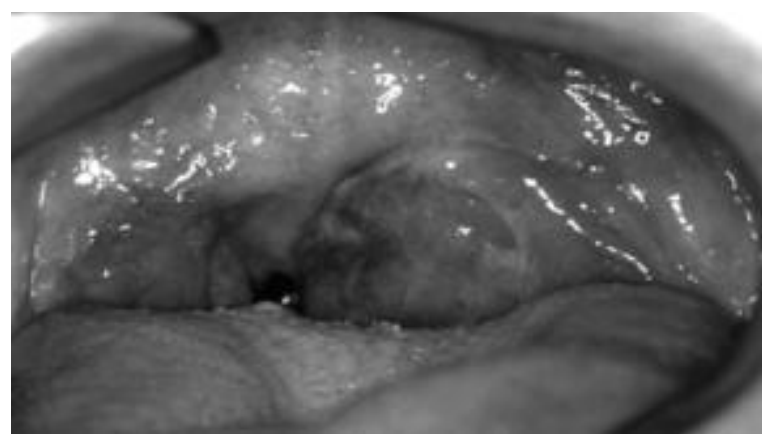

Fig. 1 : Phlégmon péri-amygdalien gauche

Tous les patients ont fait l'objet d'une ponction dans le pilier antérieur en plein bombement. Du pus franc a été retiré 55 fois soit dans $74 \%$ des cas. Dans 28 cas, le résultat bactériologique est revenu négatif ; dans 16 cas un germe a été mis en évidence (13 fois le streptocoque $\beta$ hémolytique, 3 fois un staphylocoque aureus). Dans 11 cas, le prélèvement a été effectué mais le résultat n'a malheureusement pas été récupéré.

Tous nos patients ont bénéficié d'une antibiothérapie parentérale pendant 10 jours en moyenne :

- association amoxicilline / acide clavulanique 70 cas,

- association amoxicilline / acide clavulanique et gentamycine 13 cas,

- association amoxicilline / acide clavulanique, métronidazole et gentamycine 15 cas,

- association pénicilline, métronidazole et gentamycine 3 cas.

- un malade diabétique a eu du céfotaxime concomitamment à la fosfomycine étant donné qu'il s'agissait d'une suppuration n'ayant pas répondu à l'association amoxicilline / acide clavulanique.

La durée de l'antibiothérapie a été en moyenne de 10 jours. Nous avons réalisé un drainage de la collection chez 55 malades, par incision sous anesthésie générale dans 51 cas et par ponctions évacuatrices itératives dans les 4 cas restants.

Aucune amygdalectomie à chaud n'a été effectuée.

Deux patients ont présenté une récidive du phlegmon périamygdalien, 6 semaines après le 1er épisode et avant réalisation de toute amygdalectomie. Ils ont fait l'objet d'une reprise du drainage et de l'antibiothérapie dans les 2 cas, avec une bonne évolution.

Une amygdalectomie à froid a été réalisée chez 57 patients (76\%) après un intervalle de 2 à 6 mois.

Tous nos malades ont ensuite évolué favorablement avec un recul moyen de deux ans. 


\section{DISCUSSION}

Le PPA reste le plus fréquent parmi les autres suppurations péripharyngées. (Tableau II)

\begin{tabular}{|c|c|c|c|c|c|}
\hline \multirow{2}{*}{ Auteurs } & \multirow{2}{*}{ Année } & \multicolumn{4}{|c|}{ Localisation } \\
\hline & & PPA & SPS & SRS & SRP \\
\hline Lee (4) & 2001 & - & \multicolumn{2}{|c|}{$\begin{array}{c}13 \\
(50 \%) \\
\end{array}$} & $\begin{array}{c}13 \\
(50 \%) \\
\end{array}$ \\
\hline Christine(5) & 2003 & $\begin{array}{c}8 \\
(57 \%) \\
\end{array}$ & \multicolumn{2}{|c|}{$\begin{array}{c}6 \\
(43 \%)\end{array}$} & - \\
\hline Dodds (6) & 1988 & $\begin{array}{c}42 \\
(78 \%)\end{array}$ & \multicolumn{2}{|c|}{$\begin{array}{c}3 \\
(6 \%) \\
\end{array}$} & $\begin{array}{c}9 \\
(16 \%) \\
\end{array}$ \\
\hline Notre série & 2001-07 & $\begin{array}{c}75 \\
(85 \%) \\
\end{array}$ & $(5 \%)$ & (5\%) & (3\%) \\
\hline
\end{tabular}

PPA : Phlegmon périamygdalien, SPS : suppurations préstyliennes

SRS: suppurations retrostyliennes, SRP: suppurations retro pharyngées

\section{Tableau II Prévalence comparée des suppurations péri pharyngées}

II s'agit d'une pathologie de l'adulte jeune $(7,8)$. Les âges extrêmes, rarement concernés, se présentent souvent sans fièvre et sans hypersalivation réactionnelle (2).

La présence d'angines répétées est l'antécédent le plus souvent décrit (2). Cette dernière représente la porte d'entrée principale (9), mais peut être absente dans certains cas (10)

Le diagnostic est clinique $(11,12)$, de difficulté variable. II est impératif de pouvoir examiner correctement l'oropharynx. Cela est parfois gêné par le trismus, la douleur et I'hyper-salivation. Pour cela, l'examinateur doit se mettre dans de bonnes conditions (bon éclairage, abaisse langue rigide métallique, optiques, système d'aspiration efficace). L'identification de la pathologie est facile quand il existe la triade pathognomonique trismus, œdème de la luette et bombement du pilier antérieur. Celle-ci a été constatée chez $24 \%$ des malades de CHRISTINE (13) et chez $58 \%$ de nos patients.

Des tableaux cliniques incomplets peuvent se voir. Ils risquent d'égarer le diagnostic lorsque le bombement est absent. Ces présentations peuvent être expliquées par la prescription d'une antibiothérapie non conforme aux recommandations ou par la prise d'anti-inflammatoires non stéroïdiens (AINS) masquant les premiers symptômes (2). Au moindre doute, on réalisera une ponction à l'aplomb de l'œdème au niveau de la partie supérieure du pilier antérieur qui permet, si elle ramène du pus ou même, à un stade précoce de l'évolution de la maladie, quelques simples sérosités, de confirmer le diagnostic (14). Cela a été le cas chez 55 de nos malades (73\%). Nous pouvons constater que si nous regroupons les présentations cliniques parlantes et les cas où du liquide a été aspiré, nous n'avons pas été confronté dans notre série à de gros problèmes diagnostiques.

Les PPA ont comme point de départ l'oropharynx ce qui explique la fréquence du Streptococcus bêta hémolytique A aussi bien dans notre série que dans celles de la littérature $(2,7,10,15,16,17)$.

BROOK, en utilisant des milieux de transports et de cul- tures spéciaux, a isolé une flore mixte (aéro-anaérobie) dans $76 \%$ des cas et des germes anaérobies dans 18\% des cas $(17,18)$. Dans notre étude, en raison de problèmes techniques, nous n'avons pu que suspecter la présence d'anaérobies devant la fétidité (11 cas) sans pouvoir les isoler.

Dans la plupart des cas, les germes responsables ne sont pas mis en évidence en raison de ces mêmes problèmes techniques et de l'antibiothérapie reçue avant les prélèvements $(7,19)$, justifiant à notre sens une antibiothérapie offrant une large couverture.

Dans notre étude, nous n'avons pas eu recours à l'imagerie. Elle a toutefois sa place dans cette pathologie. L'échographie intra orale peut, dans l'avenir, avoir une place dans la détection d'une collection et avoir de ce fait un intérêt diagnostic. Elle se substituerai alors à la douloureuse ponction à l'aiguille. Ceci d'autant que c'est un examen non invasif, rapide pouvant être réalisé même en présence de trismus $(20,21,22,23)$. Sa sensibilité est de $89 \%$ et sa spécificité de $100 \%$ en cas de PPA (23). Elle pourrai aussi guider la ponction, optimisant ainsi ses résultats $(5,21)$. Le scanner trouve sa place en cas de difficultés diagnostiques en présence d'un trismus très serré avec une sensibilité de $100 \%$ (23). II est aussi demandé en cas d'évolution vers une cellulite cervicale afin de juger de la diffusion de l'infection. L'IRM peut être intéressante en cas de thrombose vasculaire (24).

Nous n'avons noté aucune complication des PPA dans notre série. Celles-ci sont devenues exceptionnelles. Elles sont en général la conséquence de suppurations négligées, d'une antibiothérapie mal adaptée ou de prise d'AINS (2). La plus redoutable est la cellulite cervicale extensive associée à une médiastinite $(12,25)$.

Pour MAGDOUGALE, une monothérapie à base de pénicilline est suffisante en cas de PPA. Cet auteur ne préconise d'associer du métronidazole qu'en cas de mauvaise réponse au traitement (24). Nous pensons qu'il est préférable de viser d'emblée non seulement le streptocoque mais aussi les anaérobies et d'adapter ensuite éventuellement ce traitement aux données des résultats des prélèvements bactériologiques. L'utilisation de l'association amoxicilline / acide clavulanique concernant ces anaérobies nous semble préférable au métronidazole car la résistance a été rapporté dans 1,8\% des cas pour le premier produit contre $5 \%$ des cas pour le second (26).

La durée de cette antibiothérapie n'est pas consensuelle. La durée de 10 jours nous semble raisonnable.

La voie d'administration a toujours été pour nous la voie intraveineuse pendant toute la durée du traitement. Cette attitude nous semble maintenant excessive car autant cette voie nous semble logique à utiliser les premiers jours en raison du trismus et des difficultés d'alimentation, autant elle nous semble devoir être remplacée par la voie orale au delà.

Si la ponction revient blanche, une antibiothérapie exclu- 
sive associée à une surveillance clinique, biologique et à des ponctions répétées pour guetter la moindre récidive est logique. Dans notre série, 20 malades ont reçu une antibiothérapie exclusive avec une bonne évolution.

Certains auteurs proposent un traitement médical exclusif en cas d'une suppuration localisée de petit volume (moins de $2 \mathrm{~cm} 3$ ) et non compliquée. Une réévaluation clinique rigoureuse après 24 à 48 heures étant nécessaire (31).

Tous les auteurs (22) s'accordent à proscrire les anti inflammatoires stéroïdiens ou non car réputés favorisant l'apparition de complications.

Une fois constituées, les collections doivent être évacuées. Ponctions itératives, efficacité de $94 \%(17,27)$, drainage chirurgical, efficace dans 90 à $100 \%$ des cas $(11,17)$ sont des méthodes validées. Nous les avons utilisé toutes deux avec succès. Chacune des méthodes présente des avantages et des inconvénients. Le choix doit donc être, à notre sens, fonction des cas et de la situation. La simplicité des ponctions, pratiquées en ambulatoire sous anesthésie locale (AL), est séduisante en cas de contre indication anesthésique. Mais cette alternative présente plusieurs inconvénients. Elle est douloureuse et donc non envisageable chez l'enfant sous anesthésie locale. Elle peut être mal positionnée avec un risque de faux négatifs dans $12 \%$ des cas $(5,19,28,29,30)$.

L'incision-drainage s'effectue sous anesthésie locale ou générale. Elle présente l'avantage d'être plus radicale (en principe une seule séance suffit). Cependant, on lui reconnaît certains inconvénients par rapport à la ponction aspiration (27):

- Sa morbidité est plus importante.

- Elle ne peut être pratiquée que par un spécialiste.

- Elle est plus coûteuse.

- Le risque de dissémination de l'infection est plus important.

- Le risque de léser un vaisseau aberrant est plus important.

HIBBERT estime que le risque de récidive d'abcès périamygdalien n'est pas suffisamment important $(20 \%$ des cas) pour imposer à tous les patients une amygdalectomie (32). L'amygdalectomie à chaud reste un sujet de controverses. Elle n'est pas de pratique courante dans notre expérience.

L'amygdalectomie à froid est proposée après un délai de 1 à 6 mois. Elle est souvent rendue plus laborieuse du fait des adhérences locales souvent présentes. Pour WOLF elle est indiquée après le 2ème épisode (33). Nous pensons que ce chiffre est tout à fait arbitraire. Nous partons du principe qu'une loge amygdalienne ayant fait l'objet d'un PPA reste quoiqu'il en soit le siège de confinements et de brides qui peuvent faire le lit d'une récidive. Ainsi nous proposons systématiquement à nos patients, dés le 1 er accès de PPA, une amygdalectomie à froid après un intervalle de 2 mois.

\section{CONCLUSION}

Le phlegmon péri amygdalien est une urgence fréquente en ORL. Non traité, il expose le patient à des complications pouvant mettre en jeu le pronostic vital.

Le diagnostic, de difficulté variable, reste éminemment clinique, bien que l'échographie intra orale se fraie de plus en plus une place dans l'identification de la collection. .

Le traitement est médico-chirurgical.

Une association antibiotique à large spectre incluant les germes anaérobies, d'une durée de 10 jours, par voie intra veineuse relayée par la voie orale est le volet thérapeutique constant, indispensable. En cas de collection, le choix entre ponctions itératives et drainage par incision, souvent affaire d'école, doit à notre sens se discuter au cas par cas, chacune des techniques ayant des avantages et des inconvénients.

La meilleure attitude reste préventive, basée sur le traitement correct de toutes les portes d'entrées potentielles. 


\section{REFERENCES}

1. Kilty SJ, Gaboury I. Clinical predictors of peritonsillar abscess in adults. J Otolaryngol Head Neck Surg. 2008;37(2):165-8.

2. Pinaud V, Ballereau F, Corvec $S$ et al. Analyse de l'exposition préalable aux anti-inflammatoires et aux antibiotiques d'une cohorte de 34 patients hospitalisés au $\mathrm{CHU}$ de Nantes pour phlegmon périamygdalien. Med Mal Infect. 2009;39(12):886-90.

3. Bono G, Argo A, Zerbo S, Triolo V, Procaccianti P.Cervical necrotizing fasciitis and descending necrotizing mediastinitis in a patient affected by neglected peritonsillar abscess: a case of medical negligence. J Forensic Leg Med. 2008;15(6):391-4.

4. Lee S, Schwartz R, Babadori R. Retropharyngeal abscess: Epiglottitis of the new millennium. J Pediatr 2001; 138: 435-7.

5. Christine B.Franzese, Jon E. Isaacson, MD. Peritonsillar and Parapharyngeal Space Abscess in the Older Adult. American Journal of Otolaryngology, 2003; 24 (3): 169-173

6. Dodds B, Maniglia A. Peritonsillar and neck abscesses in the pediatric age group. Laryngoscope 1998; 98(9):956-959.

7. Apostolopous N, Nikolopoulos T, Bairamis T. Peritonsillar abscess in children. Is incision and drainage an effective management? International journal of Pediatric Otorhinolaryngology 1995; 31(2-3):129-135.

8. Laura L. Acute Infectious. Upper Airway Obstructions in children. Semin Pediatr Infect Dis, 2006; 17: 5-10.

9. Lafotte F, Martin-duverneuil N, Brunet E. Rhinopharynx et espaces profonds de la face : anatomie et applications à la pathologie. J. Neuroradiol. $1997 ; 24$ :98-107.

10. Marom T, Cinamon U, Itskoviz D, Roth $\mathrm{Y}$. Changing trends of peritonsillar abscess. Am J Otolaryngol. 2009; 22: 1-6.

11. Johnson R, Stewart M. The contemporary approach to diagnosis and management of peritonsillar abscess. Curr Opin Otolaryngol Head Neck Surg 2005;13 :157-60.

12. Johnson R, Stewart M, Wright C. An evidence-based review of the treatment of peritonsillar abscess. Otolaryngol Head Neck Surg 2003; 128:332-43.

13. Guerrier Y. Collections suppurées amygdaliennes, périamygdaliennes et péripharyngées in : Traité de technique chirurgicale ORL et cervico-faciale. Tome III. Ed MASSON. Paris 1987. p 51.

14. Barry B, Kici S, Ameline E, Bensimon JL. Suppurations péripharyngées Encyclopédie Médico-Chirurgicale 20-520-A-10.

15. Pignat J-C, Haguenauer J-P, Navailles B. Les cellulites cervicales diffuses, spontanées à anaérobies. Revue de Laryngologie 1989 -110 (2) : 141-144.

16. Sorensen J-A, Godbzelle C, Andersen N-H, Jorgensen K. Peritonsillar abscess: risk of disease in the remaining tonsil after unilateral tonsillectomy à chaud. J.Laryngol.Otol. 1991; 105, 442-444.
17. Segal N, El-Saied S, Puterman M. Peritonsillar abscess in children in the southern district of Israel. Int J Pediatr Otorhinolaryngol. 2009;73(8):1148-50.

18. Cherukuri S, Benninger MS. Use of bacteriologic studies in the out patient management of peritonsillar abscess. Laryngoscope 2002; 112: 18-20.

19. Weber AL, Siciliano A. CT and MR imaging evaluation of neck infections with clinical correlations. Radiologic Clinics of North America 2000; 38: 941-68.

20. Boyer J-C, Helenon O, Ccoste A. Apport de la tomodensitométrie dans l'exploration des suppurations cervicales. Ann. Oto-Laryngol. Chir. Cervicofac. 1994 ; $111: 59-68$.

21. Miziara ID, Koishi HU, Zonato Al . The use of ultrasound evaluation in the diagnosis of peritonsillar abscess. Rev Laryngol Otol Rhinol 2001; 122: 201- 3.

22. Wetmore R, Mahboubi S, Soyupak S. Computed tomography in the evaluation of pediatric neck infections. Otolaryngol head neck surg 1998; 119:624627.

23. Scott PM, Loftus WK, Kew J, Ahuja A, Yue V, van Hasselt CA. Diagnosis of peritonsillar infections: a prospective study of ultrasound, computerized tomography and clinical diagnosis. J Laryngol Otol. 1999;113(3):229-32

24. Macdougall G, Denholm SW. Audit of the treatment of tonsillar and peritonsillar sepsis in an ear, nose and throat unit. The journal of laryngology and otology 1995; 109: 531-533.

25. Marchal $F$, Dulguerov $P$, Lehmann W. L'abcès périamygdalien: tonsillectomie en urgence ? Ann Otolaryngol (Paris) 1995; 112: 393-398

26. Lazor JB, Cunningham M-J, Eavey R-D, Weber A-L. Comparison of computed tomography and surgical findings in deep neck infections. Otolaryngol head neck surg 1994; 111: 746-750.

27. Nagy M, Pizzuto M, Backstrom J, Brdsky L. Deep neck infections in children: a new approach to diagnosis and treatment. Laryngoscope 1997; 107: 1627-34. 28. Brook I. Microbiology of abscesses of the head and neck in children. Ann Otol Rhinol Laryngol 1987; 96: 429-433.

29. Maisl RH. Peritonsillar abscess: Tonsil antibiotic levels in patients treated by acute abscess surgery. Laryngoscope 1982; 92:80-7.

30. Marsot-dupuch .k, Portier.f . Infections cervicales et pharyngo-laryngées de l'adulte. Feuillets de Radiologie, 2003 ; 43 (6) : 508-516.

31. Ratelk K, Delis V, Oyarzabel M. Clinical differentiation of peritonsillar from abscess. Advances in Otorhinolaryngology 1994; 47: 172-5.

32. NIELSEN T-R, CLEMENT F, ANDEASSEN U-K. Mediastinitis: a rare complication of a peritonsillar abscess. The journal of laryngology and otology 1996; 110: $175-6$.

33. Wolf M, Even-Chen I, Talmi YP, Kronenberg J. The indication for tonsillectomy in children following peritonsillar abscess. Int J Pediatr Otorhinolaryngol. 1995; 31(1):43-6. 